\title{
General practitioners and necropsies
}

\section{Contacts with pathologists and coroners could change the picture}

The clinical picture during life and necropsy findings are two sides of the same coin. To look at one without the other is to see only half the picture. Proof of that assertion comes from the known discrepancy between the clinical and necropsy findings-for example, Cameron et al found that $15 \%$ of the main diagnoses and $42 \%$ of causes of death were not confirmed at necropsy and Hartveit reported that one in five diagnoses thought to be certain were found to be erroneous. ${ }^{12}$

In this issue Berlin et al report that only $20 \%$ of deaths in the community are followed by necropsies and that three quarters of those are requested by coroners for legal and not for medical reasons ( $\mathrm{p} 1080) .{ }^{3}$ Why, then, do general practitioners so rarely request necropsies when they sign about one quarter of all death certificates? Berlin et al found that in the main they favour the performance of necropsies and want to be sent the reports. They were not asked about their reluctance to request necropsies.

During discussion with small groups of general practitioners we have found two main deterrents: a belief that "I only need a postmortem if I don't know the cause of death" and anxiety about the reaction of relatives. Doctors believed that unless the relatives recognised that there are important reasons for a necropsy permission might be difficult to obtain. Indeed, Cameron et al failed to obtain permission in 35\% of deaths because of the resistance by relatives or the inadequate approach by medical staff. ${ }^{1}$ They found that female relatives were more likely to give permission than male relatives.

Berlin et al argue that more communication between general practitioners and pathologists might help. ${ }^{3}$ Initial fruitful discussions could review the number of necropsies requested by general practitioners in each hospital area, the availability of the service (which should be universal), and the resource implications. A necropsy costs roughly $£ 200$, which would presumably come from fundholding general practitioners and health commissions. If the number of necropsies rose, more staff and facilities might be needed. The next stage would be agreement on firm objectives to satisfy the needs of continuing medical education, audit, and quality control in general practice; again this should come from discussions between general practitioners and pathologists, opening up a climate of investigation and inquiry.

One issue raised by Berlin et al could be resolved quickly and easily. At present pathologists and coroners do not always send necropsy reports to general practitioners. In Southampton the coroner permits the routine distribution of these reports to general practitioners, but this is not always the case-indeed, coroners have the power to prevent the dissemination of reports. The questionnaire showed a strong demand from practitioners for these reports, and a distribution scheme organised by the family health services authority could be an important first step toward achieving collaboration between pathologists and general practitioners.

For most of us the necropsy represents finality and failure. For the patient it represents the end of life and for the doctor it represents failure of his or her efforts to improve and maintain life. Perhaps this gloom is one of the reasons why necropsies are so rarely requested. We are dealing here with matters that are fundamental and primitive. As most people, including general practitioners, have traditional and conservative attitudes, it may well be that there will be little change until more doctors make the effort to achieve closer links with their pathologist colleagues.

K B THOMAS

Department of Primary Medical Care,

University of Southampton,

Aldermoor Health Centre,

Southampton SO1 6ST

R O WELLER

Professor of neuropathology

Department of Pathology (Neuropathology),

University of Southampton, Southampton General Hospital,

Southampton SO9 $4 \mathrm{XY}$

1 Cameron HM, McGoogan E, Watson H. Necropsy: a yardstick for clinical diagnosis. $B M \mathcal{F}$ 1980;281:985-8.

2 Hartviet F. Clinical and post-mortem assessment of the cause of death. $f$ Pathol 1977;123:193-210.

3 Berlin A, Wagstaff R, Bhopal R, Spencer J. Postmortem examinations: general practitioners' knowledge, behaviour and attitudes. BMf 1994;308:1080-1.

\section{Applications of virtual reality to surgery}

\author{
Still in the prototype stage
}

Virtual reality can be defined as human interaction in an environment that is simulated by a computer. To simulate a surgical procedure interaction must be lifelike: not only must the manipulation occur in real time but the virtual anatomy must look real. Currently, the potential applications of this new technology are being explored in minimally invasive surgery, neurosurgery, and "telepresence surgery."

Technologies such as graphics workstation computers and specialised tracking devices have made it possible to build an advanced prototype of a simulator for minimally invasive surgery called the virtual clinic. ${ }^{1}$ This system uses tracking devices attached to actual surgical instruments which are inserted through trocars into a fibreglass mould of the body.
Graphic representations of the body change as the instruments are moved, and interaction is visible on a high resolution computer monitor located at the head of the virtual patient. Data produced by computed tomography and magnetic resonance imaging are used to recreate the anatomy of an actual patient. Computer manipulation allows the virtual surgical instruments to interact with the virtual tissues in a way that resembles what happens in real life. New images are created as tissues are dissected.

The benefits of a computerised training system in minimally invasive surgery include being able to reproduce surgical anomalies and emergencies to work out the best operative procedures and being able to repeat individual 
steps to improve surgical technique. Procedures can also be recorded and replayed.

In neurosurgery researchers are trying to combine live video information with three dimensional computer images of the brain to help in planning operations (W Lorensen et al, proceedings of medicine meets virtual reality II, San Diego, Jan 1994). This procedure uses off the shelf video products and a computer to combine the images of both signals. The surgeon sees a computed tomography scan or magnetic resonance imaging scan projected over the actual patient. The result is an intraoperative blend of video and computer data.

Volumetric stereotaxis is another method of planning neurosurgery (P Kelly, proceedings of medicine meets virtual reality II, San Diego, Jan 1994). This technique also uses datasets obtained with computed tomography and magnetic resonance imaging. The patient and the scan are linked by the technology of frameless stereotaxis. Magnetic field digitisers (tracking devices using magnetic forces) and computers are used to show where the instruments are. The intraoperative computer uses the information from the intraoperative microscope to update the surgeon continuously on the position of the surgical instrument. These methods of planning neurosurgical procedures are useful both preoperatively and perioperatively. They can help a surgeon to plan the best site for a skin incision, craniotomy, and brain incision. Such accuracy would minimise the injury to normal brain tissue. Perioperatively the procedure could pinpoint the site of the lesion, indicating in real time where the abnormal tissue ends and normal tissue begins. But, while these methods are promising, they have so far proved difficult to use. Hopefully, as the technology improves and becomes more user friendly neurosurgeons will be able to use these systems on patients with previously inoperable tumours.

Telepresence surgery is performed on a patient in an operating theatre containing a stereoscopic camera and a robot. At a separate location the surgical control workstation has a three-dimensional monitor the surgical input-output devices closely resembling the instruments that would be used in an operation. ${ }^{2}$ This system allows a surgeon to operate on patients in remote locations and has been developed for use in battlefields. It could, however, enable specialised surgeons to operate on patients at a distance.

Although all of these systems are still being developed, they hold great promise for future training and planning and performance of surgical procedures.

Cine'-Med is researching and developing virtual clinic surgical simulation.

Cine'-Med, KEVIN T McGOVERN

127 Main Street North,

Woodbury,

CT 06798, USA

1 McGovern KT, McGovern LT. The virtual clinic, a virtual reality surgical clinic. Virtual Reality World 1994;Mar-Apr:41-4.

2 Satava RM. The role of virtual reality in medicine of the 21st Century. Virtual Reality Systems 1993;1(no 2):64-7.

\title{
At what blood alcohol concentration should drink-driving be illegal?
}

\author{
Something lower than $17 \cdot 4 \mathrm{mmol} /(0 \cdot 8 \mathrm{~g} / \mathrm{l})$
}

Legislators decide the maximum legal blood alcohol concentration for drivers, ' but their decisions should be informed by the best available scientific evidence, and here researchers have a role.

Data from case-control studies of accident involvement and experimental studies of alcohol's effects on driving skills were considered before Britain introduced its blood alcohol limit in 1967. ${ }^{2}$ Results from the Grand Rapids case-control study ${ }^{3}$ and the Medical Research Council's research on simulated driving ${ }^{4}$ were interpreted as supporting the limit of $17 \cdot 4 \mathrm{mmol} / \mathrm{l}$. Different analyses of the data, however, could have supported a lower legal limit of $10 \cdot 9-13 \cdot 0 \mathrm{mmol} / 1 .^{56}$

Reviewing data from several case-control studies, the National Research Council of the United States concluded that focusing on the risk of crash responsibility (as opposed to crash involvement) or alternatively controlling for the driver's drinking experience supported legal limits below $17 \cdot 4$ mmol/ $/ .^{6}$ It decided that "for large groups of drivers the responsibility for crashes increases when the driver judged culpable for the crash has a blood alcohol concentration greater than $8.7 \mathrm{mmol} / 1$. ." When the case control data were controlled for experience with alcohol the risk of crash involvement began increasing at the lowest blood alcohol concentrations measured.

Behavioural studies suggest that driving related skills are significantly impaired at blood alcohol concentrations below $10.9 \mathrm{mmol} / \mathrm{l}$ and that little evidence exists for a threshold below which driving related skills are unimpaired. ${ }^{167}$ In another recent review of behavioural studies, Kruger concluded that driving skills were significantly impaired at blood alcohol concentrations between 6.5 and $17.4 \mathrm{mmol} / 1$, depending on the task's demands and complexity. ${ }^{8}$ Kruger believed, however, that "a general endangerment probably only takes effect at blood alcohol concentrations at or above $15 \cdot 2$ to $17 \cdot 4$ $\mathrm{mmol} / \mathrm{h}$." This probably reflects the fact that experienced drinkers with good psychomotor skills show relatively little impairment, even at blood alcohol concentrations of nearly $21.7 \mathrm{mmol} / 1$, on simple driving tasks. ${ }^{9}$

Furthermore, Kruger suggested that "additional arguments" would be needed to justify a limit lower than $15 \cdot 2$ $\mathrm{mmol} / \mathrm{l}$. Probably the strongest additional arguments come from the falls in drink-driving behaviour and related casualties that lowering the legal limit may achieve.

A recent report examined the effect of reducing the limit from $17.4 \mathrm{mmol} / 1$ to $10.9 \mathrm{mmol} / 1$ in the Australian Capital Territory. ${ }^{10}$ Among drivers sampled at random road blocks significantly fewer had blood alcohol concentrations over $32 \cdot 6$ $\mathrm{mmol} / \mathrm{l}$ and between 10.9 and $17.4 \mathrm{mmol} / \mathrm{l}$ than before the law was changed.

In addition, the proportion of drivers with blood alcohol concentrations above $17.4 \mathrm{mmol} / \mathrm{l}$ involved in accidents fell by one third, and these effects seemed sustained for at least 12 months after the change. The overall number of accidents fell by $6 \%$. This study suggested that behavioural change is possible without increasing the demands made on the police -one of the factors that would militate against change.

Whether a limit of $10.9 \mathrm{mmol} / \mathrm{l}$ would substantially affect the more serious offenders is difficult to judge. A lower legal limit might not deter those current drink-drivers who believe 